International Journal of Modern Physics B

(C) World Scientific Publishing Company

\title{
Quantum limit of measurement and the projection postulate
}

\author{
S.A. Gurvitz \\ Department of Particle Physics, Weizmann Institute of Science, Rehovot 76100, Israel \\ shmuel.gurvitz@weizmann.ac.il
}

\begin{abstract}
We study an electrostatic qubit monitored by a point-contact detector. Projecting an entire qubit-detector wave function on the detector eigenstates we determine the precision limit for the qubit measurements, allowed by quantum mechanics. We found that this quantity is determined by qubit dynamics as well as decoherence, generated by the measurement. Our results show how the quantum precision limit can be improved by a proper design of a measurement procedure.
\end{abstract}

Keywords: electrostatic qubit; point-contact detector; projection postulate; decoherence; precision limit

Rapid experimental progress in monitoring of single quantum systems renewed the interest in the quantum mechanical limitation of measurement accuracy ${ }^{1}$. This limitation is originated from the uncertainty relation between observables of a measured microscopic system, the so-called standard quantum limit of measurement ${ }^{2}$. Yet, a single quantum system is not observed directly, but through the interaction with a measurement device (detector). This implies that the quantum limit of measurement is not related to a measured system only, but rather to an entire system, including detector ${ }^{2}$.

A measurement in the quantum mechanical formalism corresponds to projection of the wave function of an entire system on eigenstates of the detector, accessible by an observer. This is the so-called projection postulate ${ }^{3}$, analogues to the Bayes principle in any probabilistic description. Since the detector and the measured system are interacting, the above projection on the detector states affects the measured microscopic system. If the latter is projected on one of its own states, then the microscopic system can be measured with any accuracy. However, if this system is projected on the superposition of its states, the measurement cannot be precise. Its accuracy is given by a size of the corresponding wave packet.

In this Letter we apply the above described procedure for a determination of the precision limit of quantum measurements. As an example we take an electron trapped inside a double-dot (electrostatic qubit) and continuous monitored by a point-contact detector ${ }^{4}$. The total system can be treated entirely quantum mechanically, although the detector represents a macroscopic (mesoscopic) device ${ }^{5}$. This allows us to obtain the detector eigenstates and then to perform projections of the total wave function on these eigenstates without any additional assumptions. 
2 S.A. Gurvitz

Consider a qubit, represented by an electron in the double-dot, placed near a point-contact that separated two reservoirs (the point-contact detector), Fig. 1. When the first dot, which is far away from to the point-contact, is occupies (Fig. 1a), the current through the point-contact is $I_{1}$. If the second dot, close to the point contact, is occupied (Fig. 1b), the current decreases $\left(I_{2}<I_{1}\right)$ due to the electrostatic repulsion. The entire system can be described by the following tunneling Hamiltonian $^{5}: H=H_{P C}+H_{D D}+H_{i n t}$, where

$$
\begin{aligned}
\mathcal{H}_{P C} & =\sum_{l} E_{l} a_{l}^{\dagger} a_{l}+\sum_{r} E_{r} a_{r}^{\dagger} a_{r}+\sum_{l, r} \Omega_{l r}\left(a_{l}^{\dagger} a_{r}+H . c .\right), \\
\mathcal{H}_{D D} & =E_{1} c_{1}^{\dagger} c_{1}+E_{2} c_{2}^{\dagger} c_{2}+\Omega\left(c_{2}^{\dagger} c_{1}+c_{1}^{\dagger} c_{2}\right), \\
\mathcal{H}_{\text {int }} & =\sum_{l, r} \delta \Omega_{l r} c_{2}^{\dagger} c_{2}\left(a_{l}^{\dagger} a_{r}+H . c .\right),
\end{aligned}
$$

and $\delta \Omega_{l r}=\Omega_{l r}^{\prime}-\Omega_{l r}$. Here $a_{l, r}^{\dagger}\left(a_{l, r}\right)$ is the creation (annihilation) operator for an electron at the level $l$ or $r$ in the left or right reservoir, and $c_{1,2}^{\dagger}\left(c_{1,2}\right)$ is the same operator for the electron inside the double-dot. $\Omega_{l r}$ is the hopping amplitude between the states $l$ and $r$ of the reservoirs, and $\Omega$ is the hopping amplitude between the states $E_{1}$ and $E_{2}$ of the qubit. For simplicity we consider electrons as spin-less fermions. The interaction term $H_{\text {int }}$ generates variation of the hopping amplitude, $\delta \Omega_{l r}=\Omega^{\prime}{ }_{l r}-\Omega_{l r}$, resulting in a decrease of the detector current from $I_{1}$ to $I_{2}$, Fig. 1.
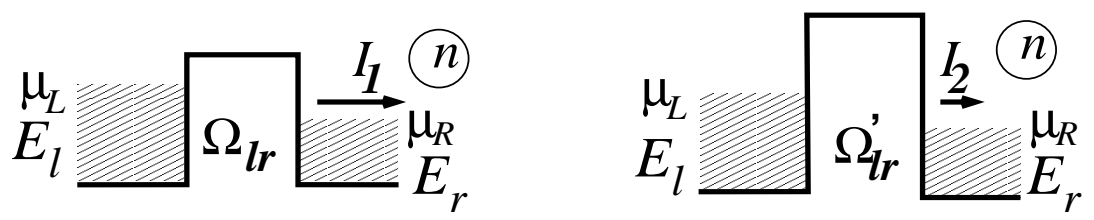

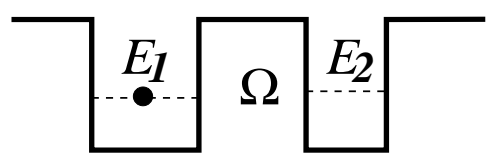

(a)

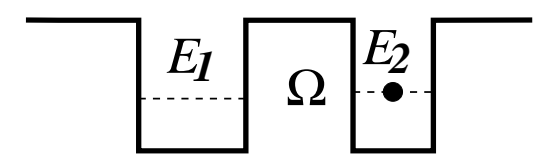

(b)

Fig. 1: The electrostatic qubit monitored by the point-contact detector. The detector current decreases when the left dot of the qubit is occupied. Here $n$ denotes the number of electrons which have arrived at the right reservoir by time $t$.

The wave function describing the entire system can be written as

$$
|\Psi(t)\rangle=\left[b_{1}(t) c_{1}^{\dagger}+\sum_{l, r} b_{1 l r}(t) c_{1}^{\dagger} a_{r}^{\dagger} a_{l}+\sum_{\substack{l<l^{\prime} \\ r<r^{\prime}}} b_{1 l l^{\prime} r r^{\prime}}(t) c_{1}^{\dagger} a_{r}^{\dagger} a_{r^{\prime}}^{\dagger} a_{l} a_{l^{\prime}}\right.
$$




$$
\left.+b_{2}(t) c_{2}^{\dagger}+\sum_{l, r} b_{2 l r}(t) c_{2}^{\dagger} a_{r}^{\dagger} a_{l}+\sum_{\substack{l<l^{\prime} \\ r<r^{\prime}}} b_{2 l l^{\prime} r r^{\prime}}(t) c_{2}^{\dagger} a_{r}^{\dagger} a_{r^{\prime}}^{\dagger} a_{l} a_{l^{\prime}}+\cdots\right]|0\rangle,
$$

where $b_{\alpha}(t)$ is the amplitude of finding the system in the state $\alpha$ determined by the corresponding creation and annihilation operators. These operators act on the initial ("vacuum") state, $|0\rangle$. For simplicity we assume that the reservoirs are initially at zero temperature and filled with electrons up to the Fermi energies $\mu_{L, R}$, respectively. All the amplitudes $b_{\alpha}(t)$ can be obtained from the time-dependent Schrödinger equation, $\partial_{t}|\Psi(t)\rangle=H|\Psi(t)\rangle$.

Now we project the total wave function (4) on the detector eigenstates. There exists an uncertainty however, about a choice of the eigenstates, since different detector variables can be recorded ${ }^{6}$. In fact, the point-contact detector is not recorded directly, but via another readout device ("pointer"). The latter can single out a particular set of the eigenstates through a coupling with the corresponding detector variables. Yet, in this work we do not extend our system by including such a pointer in the Schrödinger equation. We assume instead that the detector states are directly accessible to an "observer". Let us examine different alternatives of the measurement.

Consider first the measurement of number of electrons $(n)$ in the right reservoir (the accumulated charge), Fig. 1. This implies that the wave function, $|\Psi(t)\rangle$, Eq. (4), is projected on the eigenstates of the operator $\hat{N}=\sum_{r} a_{r}^{\dagger} a_{r}$, which can be written as

$$
|n\rangle=\underbrace{a_{r_{1}}^{\dagger} a_{r_{2}}^{\dagger} \cdots a_{r_{n}}^{\dagger}}_{n}|0\rangle .
$$

(Note, that the state $|n\rangle$ is strongly degenerate: $|n\rangle \equiv|n, \alpha\rangle$, where $\alpha$ corresponds to a particular configuration of $n$ electrons in the collector). Thus, $|\Psi(t)\rangle \rightarrow$ $\mathcal{N}^{-1 / 2} \hat{P}_{n}|\Psi(t)\rangle$, where $\hat{P}_{n}=\sum_{\alpha}|n, \alpha\rangle\langle n, \alpha|$ is a projection operator and $\mathcal{N}$ is a normalization factor. One finds from Eqs. (4) and (5) that

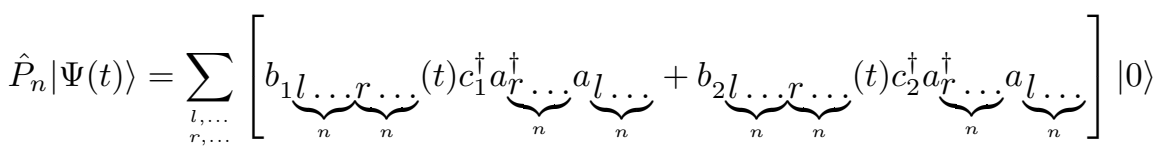

where the corresponding normalization factor $\mathcal{N}=\sum_{l, \ldots, r, \ldots}\left[\left|b_{1 l \ldots . r}(t)\right|^{2}+\left|b_{2 l \ldots r}(t)\right|^{2}\right]$ is a probability of finding $n$ electrons in the collector by time $t$. Eq. (6) shows that the measurement leaves the qubit in a linear superposition of its two states. Therefore one cannot determine the state of the qubit by measuring the number of electrons in the right reservoirs.

Consider now the measurement of electric current in the right reservoir. The latter is given by a commutator of $\hat{N}$ with the total Hamiltonian of the system, 
$\hat{I}=i[H, \hat{N}]$ (we choose $e=1$ ). Using Eqs. (1)-(3) we obtain

$$
\hat{I}=i \sum_{l, r}\left(\Omega_{l r}+\delta \Omega_{l r} c_{2}^{\dagger} c_{2}\right)\left(a_{l}^{\dagger} a_{r}-a_{r}^{\dagger} a_{l}\right) \equiv \sum_{l, r} \hat{I}_{l r}
$$

The eigenstates of the energy resolved-current operator, $\hat{I}_{l r}\left|I_{l r}^{ \pm}(q)\right\rangle=I_{l r}^{ \pm}(q)\left|I_{l r}^{ \pm}(q)\right\rangle$ are

$$
\left|I_{l r}^{ \pm}(q)\right\rangle=\frac{1}{\sqrt{2}}\left(a_{r}^{\dagger} a_{l} \pm i\right) c_{q}^{\dagger}|0\rangle,
$$

where $q=1,2$ denotes the qubit state, $c_{q}^{\dagger}|0\rangle$ and $I_{l r}^{ \pm}(q)= \pm\left[\Omega_{l r}+(q-1) \delta \Omega_{l r}\right]$. Respectively, the eigenstates of the total current, $|I\rangle$, are given by a product of $\left|I_{l r}^{ \pm}\right\rangle$.

It follows from Eqs. (7), (8) that by measuring the energy-resolved current $\hat{I}_{l r}$ (or the total current, $\hat{I}$ ), one projects the wave function (4) on a certain state of the qubit, corresponding to an observed value of the current. This implies that the qubit position can be determined with any accuracy, in principle, by monitoring directly the detector current (via its magnetic field ${ }^{7}$ ).

If, however, such a direct measurement of the detector current cannot be performed, one can determine it indirectly, via a variation of the collector charge. Let us assume that we recorded $n_{0}$ electrons in the collector at time $t$. As a result, the entire system is projected to the state $\left|n_{0}\right\rangle:|\psi(t)\rangle \rightarrow \hat{P}_{n_{0}}|\psi(t)\rangle$, Eq. (6), which is an eigenstate of the operator $\hat{N}$, Eq. (5). (We omitted the index of degeneracy $\alpha$ ). Next we detect the accumulated charge $n$ at the time $t+\Delta t$. The final state of the detector (up to the normalization factor) is

$$
\hat{P}_{n} e^{-i H \Delta t} \hat{P}_{n_{0}}|\Psi(t)\rangle=|n\rangle\left\langle\varphi_{n}(\Delta t) \mid n_{0}\right\rangle\left\langle n_{0} \mid \Psi(t)\right\rangle,
$$

where $\left|\varphi_{n}(\Delta t)\right\rangle=\exp (i H \Delta t)|n\rangle$ is an eigenstate of the operator $\hat{N}(\Delta t)=$ $\exp (i H \Delta t) \hat{N} \exp (-i H \Delta t)$, corresponding to an eigenvalue $n$. This operator can be expanded in powers of $\Delta t$

$$
\hat{N}(\Delta t)=\hat{N}+\hat{I} \Delta t+i[H, \hat{I}] \frac{(\Delta t)^{2}}{2}+\cdots,
$$

where the current $\hat{I}$ is given by Eq. (7). Thus the time-dependent operator $\hat{N}(\Delta t)$ includes the qubit position operator, $c_{q}^{\dagger} c_{q}$, in contrast with $\hat{N} \equiv N(0)$. As a result the projection on the eigehstates of $\hat{N}(\Delta t)$, Eq. (9), could determine the qubit position.

Let us take small $\Delta t$ ("measurement time") in Eq. (10) such that $\hat{N}(\Delta t) \simeq$ $\hat{N}+\hat{I} \Delta t$. If $\hat{N}$ and $\hat{I}$ commute, then the eigenstates of $\hat{N}(\Delta t)$ would be a product of eigenstates of these operators: $\left|\varphi_{n}(\Delta t)\right\rangle=\left|n^{\prime}, I(q)\right\rangle$, where $q=\{1,2\}$ denotes the qubit state and $n=n^{\prime}+I(q) \Delta t$. It follows from Eq. (9) that $n^{\prime}=n_{0}$ and $I(q) \equiv I_{\Delta n}(q)=\Delta n / \Delta t$, where $\Delta n=n-n_{0}$. As a result the qubit is projected in the state $q$ corresponding to the variation of the collector charge, $\Delta n$.

In fact, the operators $\hat{N}$ and $\hat{I}$ do not commute. In this case it is only the average current, $\bar{I}_{\Delta n}=\overline{\Delta n} / \Delta t$ which can be determined from ensemble measurements of 
$\Delta n$, where its dispersion, $\delta I_{\Delta n}=\left[\overline{(\Delta n)^{2}}-(\overline{\Delta n})^{2}\right]^{1 / 2} / \Delta t$, diverges as $(\Delta t)^{-1 / 2}$ for $\Delta t \rightarrow 0$. The latter restricts the accuracy of the qubit measurements, respectively.

The measurement accuracy, however, increases by increasing the measurement time, since $\delta I_{\Delta n}(q) \rightarrow 0$ for $\Delta t \rightarrow \infty^{8}$. Yet, this can be done only if the qubit is not "moving", (i.e. the hopping amplitude $\Omega=0$, Fig. 1 ). Then $[H, \hat{I}]=0$, so that the higher order terms in the expansion (10) vanish. If it is not the case $(\Omega \neq 0)$, the current it driven by the qubit, so that $[H, \hat{I}]$ and the higher order commutators in Eq. (10) are not zero. The average contribution from these terms to $I_{\Delta n}$, which we denote as $\delta_{1} I_{\Delta n}(\Delta t)$, increase with $\Delta t$. This suggests that the quantum limit of the qubit measurement is determined by the optimal measurement time $(\Delta t)$ which minimizes the total error,

$$
\left[\delta_{2} I_{\Delta n}(\Delta t)\right]^{2}=\left[\delta I_{\Delta n}(\Delta t)\right]^{2}+\left[\delta_{1} I_{\Delta n}(\Delta t)\right]^{2} .
$$

In order to perform this procedure we introduce the density matrix $\sigma_{q q^{\prime}}^{(n)}(t)=$ $\left\langle n, q^{\prime} \mid \Psi(t)\right\rangle\langle\Psi(t) \mid n, q\rangle$, where the wave function $|\Psi(t)\rangle$ is given by Eq. (4). It was demonstrated in $^{5,9}$ that for large large bias voltage, $V=\mu_{L}-\mu_{R}$ (Fig. 1), the time-dependent Schrödinger equation, $\partial_{t}|\Psi(t)\rangle=H|\Psi(t)\rangle$, can be reduced to the following Bloch-type rate equations for the density matrix $\sigma_{q q^{\prime}}^{(n)}(t)$ by assuming weak energy dependence of the transition amplitudes $\left(\Omega_{l r}=\bar{\Omega}, \Omega_{l r}^{\prime}=\bar{\Omega}^{\prime}\right)^{5}$,

$$
\begin{aligned}
& \dot{\sigma}_{11}^{(n)}=-D_{1} \sigma_{11}^{(n)}+D_{1} \sigma_{11}^{(n-1)}+i \Omega\left(\sigma_{12}^{(n)}-\sigma_{21}^{(n)}\right) \\
& \dot{\sigma}_{22}^{(n)}=-D_{2} \sigma_{22}^{(n)}+D_{2} \sigma_{22}^{(n-1)}-i \Omega\left(\sigma_{12}^{(n)}-\sigma_{21}^{(n)}\right) \\
& \dot{\sigma}_{12}^{(n)}=i\left(E_{2}-E_{1}\right) \sigma_{12}^{(n)}+i \Omega\left(\sigma_{11}^{(n)}-\sigma_{22}^{(n)}\right)-\frac{D_{1}+D_{2}}{2} \sigma_{12}^{(n)}+\left(D_{1} D_{2}\right)^{1 / 2} \sigma_{12}^{(n-1)}(14)
\end{aligned}
$$

where $D_{1,2}=T_{1,2} V$ and $T_{1,2}$ is the transmission probability of the barrier: $T_{1}=$ $(2 \pi)^{2} \bar{\Omega}^{2} \rho_{L} \rho_{R}$ and $T_{2}=(2 \pi)^{2}\left(\bar{\Omega}^{\prime}\right)^{2} \rho_{L} \rho_{R}$, where $\rho_{L, R}$ is the density of states in the left (right) reservoir, Fig. 1.

Solving Eqs. (12)-(14) one can find all quantities needed for evaluation of $\delta I_{\Delta n}$ and $\delta_{1} I_{\Delta n}$. For instance, in order to evaluate the average value of $\Delta n$ and its dispersion we have to solve these equations with the initial condition $n=n_{0}$. It follows from Eqs. (12)-(14) that the density matrix $\sigma_{q q^{\prime}}^{(n)}(t)$ depends only on $\Delta n=n-n_{0}$. Thus we can take $n_{0}=0$ and $\Delta n=n$. Then the average values $\overline{\Delta n}=\bar{n}$ and $\overline{(\Delta n)^{2}}=\overline{n^{2}}$ are given by

$$
\begin{gathered}
\bar{n}(t)=\sum_{n} n P_{n}(t)=\bar{n}_{11}(t)+\bar{n}_{22}(t), \\
\overline{n^{2}}(t)=\sum_{n} n^{2} P_{n}(t)=\overline{n_{11}^{2}}(t)+\overline{n_{22}^{2}}(t),
\end{gathered}
$$

where $P_{n}(t)=\sigma_{11}^{(n)}(t)+\sigma_{22}^{(n)}(t)$ is the probability of finding $n$ electron in the collector. Multiply Eqs. (12)-(14) by $n$ and sum over $n$ one finds

$$
\begin{aligned}
& \dot{\bar{n}}_{11}=D_{1} \sigma_{11}+i \Omega\left(\bar{n}_{12}-\bar{n}_{21}\right) \\
& \dot{\bar{n}}_{22}=D_{2} \sigma_{22}-i \Omega\left(\bar{n}_{12}-\bar{n}_{21}\right)
\end{aligned}
$$




$$
\dot{\bar{n}}_{12}=i\left(E_{2}-E_{1}\right) \bar{n}_{12}+i \Omega\left(\bar{n}_{11}-\bar{n}_{22}\right)-\frac{\Gamma_{d}}{2} \bar{n}_{12}+\left(D_{1} D_{2}\right)^{1 / 2} \sigma_{12},
$$

where $\sigma_{q q^{\prime}}(t)=\sum_{n} \sigma_{q q^{\prime}}^{(n)}(t)$ is the qubit density matrix, and $\Gamma_{d}=\left(\sqrt{D_{1}}-\sqrt{D_{2}}\right)^{2}$ is the decoherence rate ${ }^{5}$.

Similarly multiplying Eqs. (12)-(14) by $n^{2}$ and sum over $n$ one obtains

$$
\begin{aligned}
& \dot{\dot{n_{11}^{2}}}=2 D_{1} \bar{n}_{11}+D_{1} \sigma_{11}+i \Omega\left(\overline{n_{12}^{2}}-\overline{n_{21}^{2}}\right) \\
& \dot{n_{22}^{2}}=2 D_{2} \bar{n}_{22}+D_{2} \sigma_{22}-i \Omega\left(\overline{n_{12}^{2}}-\overline{n_{21}^{2}}\right) \\
& \dot{\check{n_{12}^{2}}}=i\left(E_{2}-E_{1}\right) \overline{n_{12}^{2}}+i \Omega\left(\overline{n_{11}^{2}}-\overline{n_{22}^{2}}\right)-\frac{\Gamma_{d}}{2} \overline{n_{12}^{2}}+\left(D_{1} D_{2}\right)^{1 / 2}\left(2 \bar{n}_{12}+\sigma_{12}\right) .
\end{aligned}
$$

The qubit density matrix $\sigma_{q q^{\prime}}(t)$ can be easily found from Eqs. (12)-(14) by tracing it over $n$,

$$
\begin{aligned}
& \dot{\sigma}_{11}=i \Omega\left(\sigma_{12}-\sigma_{21}\right) \\
& \dot{\sigma}_{12}=i \epsilon_{21} \sigma_{12}+i \Omega\left(2 \sigma_{11}-1\right)-\frac{\Gamma_{d}}{2} \sigma_{12},
\end{aligned}
$$

and $\sigma_{22}(t)=1-\sigma_{11}(t)$. This quantity, $\sigma_{q q^{\prime}}(t)$, determines the detector average current, $\bar{I}(t)=\langle\Psi(t)|\hat{I}| \Psi(t)\rangle$. Indeed, it follows from Eqs. (4), (7) that

$$
\bar{I}(t)=D_{1} \dot{\bar{n}}_{11}(t)+D_{2} \dot{\bar{n}}_{22}(t)=D_{2}+\Delta D \sigma_{11}(t),
$$

where $\Delta D=D_{1}-D_{2}$ is an average "signal". Obviously, $\bar{I}(\Delta t)=\bar{n} / \Delta t \equiv \bar{I}_{n}(\Delta t)$ for small $\Delta t$. The dispersion of $I_{n}(\Delta t)$ can be found from Eqs. (16), (20)-(22),

$$
\left(\delta I_{n}\right)^{2}=\overline{n^{2}}(\Delta t) /(\Delta t)^{2}-\bar{I}_{n}^{2} \simeq I_{n}(0) / \Delta t .
$$

As expected, $\delta I_{n}(\Delta t)$ diverges as $1 / \sqrt{\Delta t}$ for $\Delta t \rightarrow 0$.

Let us evaluate the contribution from higher order terms in the expansion (10), which generate variation of the average current, $\delta_{1} I_{n}$. (In our case this variation is produced by the qubit only). Therefore, one can write $\delta_{1} I_{n}(\Delta t)=\left|\bar{I}_{n}(\Delta t)-\bar{I}_{n}(0)\right|$. Using Eq. (25) we evaluate this quantity as

$$
\delta_{1} I_{n}=\Delta D\left|\sigma_{11}(\Delta t)-\sigma_{11}(0)\right|,
$$

where $\sigma_{11}(t)$ is obtained from Eqs. (23)-(24). For instance, for aligned levels, $E_{1}=$ $E_{2}$, and $\sigma_{11}(0)=1$,

$$
\delta_{1} I_{n}=\frac{\Delta D}{2}\left|e^{-\frac{\Gamma_{d}}{4} \Delta t}[\cos (\omega \Delta t)+\eta \sin (\omega \Delta t)]-1\right|,
$$

where $\eta=\Gamma_{d} / 4 \omega$ and $\omega=2 \Omega \sqrt{1-\left(\Gamma_{d} / 8 \Omega\right)^{2}}$ is the Rabi frequency. As expected, $\delta_{1} I_{n} \rightarrow 0$ for $\Omega \rightarrow 0$.

Eqs. (26) and (28) allow us to evaluate the optimal $\Delta t$ by minimizing $\left[\delta_{2} I_{n}(\Delta t)\right]^{2}$, Eq. (11). We take for simplicity $\Delta D \ll D$, where $D=\left(D_{1}+D_{2}\right) / 2$. As a result $\Gamma_{d} \simeq(\Delta D)^{2} / 4 D$. We first consider weak distortion of the qubit, $\Gamma_{d} / 8 \ll \Omega$. Then 
expanding Eq. (28) in powers of $\Delta t$ we easily obtain for the optimal measurement time and for the corresponding measurement limit

$$
\Delta t=\frac{1}{2 \Omega}\left(\frac{2 \Omega}{\Gamma_{d}}\right)^{1 / 5}, \quad\left(\delta_{2} I_{n}\right)^{2}=\frac{5 D \Omega}{2}\left(\frac{\Gamma_{d}}{2 \Omega}\right)^{1 / 5} .
$$

In order to observe Rabi oscillations of the qubit in a single measurement run one needs $\delta_{2} I_{n} \ll \Delta D$, at least. It follows from Eq. (29) that this condition corresponds to $\Omega \ll 2 \Gamma_{d}$. This, however cannot be combined with the condition of weak qubit distortion, used in Eq. (29). Hence, one cannot observe Rabi oscillations in a single run, but only in an ensemble average of different runs.

Consider now large decoherence limit, $\Gamma_{d} / 8 \gg \Omega$. Then the qubit is strongly affected by the detector. As a result, the electron stays in the same dot for a long time, $t \sim \Gamma_{d} / 8 \Omega^{2}$ ("quantum Zeno" effect). Indeed, one finds from Eqs. (23)-(24) that $\sigma_{11}=\left[1+\exp \left(-8 \Omega^{2} t / \Gamma_{d}\right)\right] / 2$ for $t \gg 1 / \Gamma_{d}$. Respectively, the optimal measurement time and the measurement limit are given by

$$
\Delta t=\frac{1}{4 \Omega}\left(\frac{\Gamma_{d}}{2 \Omega}\right)^{1 / 3}, \quad\left(\delta_{2} I_{n}\right)^{2}=6 D \Omega\left(\frac{2 \Omega}{\Gamma_{d}}\right)^{1 / 3} .
$$

In contrast with the previous case, Eq. (29), the measurement time $\Delta t$ increases with $\Gamma_{d}$. This is not surprising since large decoherence generated by the detector, localizes the qubit for a long time. Therefore it behaves as a static one so that the measurement time increases.

It follows from our arguments that the quantum precision limit depends on a particular set of the detector observables which the total wave function is projected on. In this Letter we discussed two alternative sets related to charge and current states of the point-contact detector. It was demonstrated that single projection on charge states cannot measure the qubit state. However, two consecutive projections of the entire system on the charge states can measure the qubit state, but only with a limited accuracy. On the other hand, direct projection on the current state of the detector can determine the qubit state with absolute precision, in principle. If this could be realized, one would arrive to the Zeno paradox ${ }^{10}$, i.e. to complete freezing of a system as a result of continuous measurement.

This shows a necessity of including a "pointer" in the total Hamiltonian coupled with current states of the detector (von Neumann hierarchy ${ }^{3}$ ). In this case two consecutive projections of the total wave function on the pointer states, used for a determination of the detector current, would restrict the measurement accuracy in a total analogy with the previous case. If the pointer is coupled with charge states of the detector, it obviously cannot decrease the quantum measurement limit found in our calculations. We assume also that the pointer cannot essentially increase this limit, since otherwise the von Neumann hierarchy of measurements would not converge. This problem, however needs a special attention.

Our final results on quantum limit of measurement involved only average quantities, which were obtained without any explicit resort to the projection postu- 
late. Indeed, the latter is related only to a single measurement. Nevertheless, as we demonstrated in this Letter, the use of the projection postulate was very useful in a determination of quantum limit of measurement. In particular, it clearly displayed the detector variables which would allow us to measure a microscopic system with maximal accuracy. Such variables are usually represent commutators (time derivatives) of operators describing the detector states.

An appropriate choice of this variable depends of a particular measurement apparatus. For instance, for a single electron transistor (SET) detector, one needs to use the second commutator ("acceleration") of the accumulated charge. In contrast with the point-contact detector, the projection to current states of the SET would not determine the qubit state. The measurement of the charge "acceleration" can be very useful if the corresponding operator would commute with the charge operator. In this case one can design an appropriate procedure of projecting on the charge states at different times which would diminish the quantum measurement limit. This however must be a subject of a separate investigation.

\section{References}

1. R. Ruskov, K. Schwab, and A.N. Korotkov, Phys. Rev. B71, 235407 (2005).

2. V.B. Braginsky and F.Ya. Khalili, Quantum measurement (Cambridge Univ. Press, Cambridge, UK, 1992).

3. J. von Neumann, Mathematical Foundations of Quantum Mechanics (Princeton University Press, Princeton, 1955).

4. T. Hayashi, T. Fujisawa, H.D. Cheong, Y.H. Jeong, and Y. Hirayama, Phys. Rev. Lett. 91, 226804 (2003); J.R. Petta, A.C. Johnson, C.M. Marcus, M.P. Hanson, and A.C. Gossard, Phys. Rev. Lett. 93, 186802 (2004).

5. S.A. Gurvitz, Phys. Rev. B56, 15215 (1997).

6. W.H. Zurek, Rev. Mod. Phys. 75, 715 (2003).

7. L.S. Levitov and H. Lee and G.B. Lesovik, J. Math. Phys. 37, 4845 (1996).

8. In this limit $\hat{N}(\Delta t)=\hat{N}+\hat{I} \Delta t$ is dominated by the second term. As a result, the measurement of $\Delta n$ determines the current $I$, and therefore the qubit is projected in one of its states.

9. S.A. Gurvitz and Ya.S. Prager, Phys. Rev. B B53, 15932 (1996); S.A. Gurvitz, Phys. Rev. B57, 6602 (1998).

10. B. Misra and E.C.G. Sudarshan, J. Math. Phys. 18 (1977). 